\section{Drosophila argonaute-2 is required early in embryogenesis for the assembly of centric/ centromeric heterochromatin, nuclear division, nuclear migration, and germ-cell formation}

\author{
Girish Deshpande, Gretchen Calhoun, and \\ Paul Schedl ${ }^{1}$ \\ Department of Molecular Biology, Princeton University, \\ Princeton, New Jersey 08540, USA
}

The RNA-induced silencing complex (RISC) or the RISC complex mediates RNAi and is comprised of proteins belonging to the dicer and Argonaute family proteins. Here we show that Argonaute-2 (ago-2) is required for proper nuclear migration, pole cell formation, and cellularization during the early stages of embryonic development in Drosophila. We have traced these defects back to the nuclear division cycles. Unlike wild type, nuclear division is asynchronous in ago-2 embryos and there are defects in chromosome condensation, nuclear kinesis, and assembly of spindle apparatus. The aberrations in the nuclear division cycle are correlated with defects in the formation of centric/centromeric heterochromatin and point to a failure in the assembly of functional centromeres.

Supplemental material is available at http://www.genesdev.org.

Received March 21, 2005; revised version accepted May 26, 2005.

The first several hours of Drosophila embryonic development occur as a syncytium of nuclei without any cells (Baker et al. 1993; Sullivan et al. 1993). After the fusion of the male and female pronuclei, there is a period of rapid, highly synchronous nuclear division in the central region of the embryo. During cycles 8 and 9, the nuclei migrate toward the cortex of the embryo by a microtubule-dependent mechanism (Sullivan et al. 1993; von Dassow and Schubiger 1994). Nuclei entering the posterior pole plasm induce the formation of buds that protrude from the surface of the embryo (for review, see Williamson and Lehmann 1996). The nuclei in these polar buds, together with the pole plasm, cellularize to form the pole cells, the precursors of the germline. The remaining nuclei reach the periphery of the embryo dur-

[Keywords: Argonaute-2; actin-myosin cytoskeleton; centromere; heterochromatin; nuclear division; spindle apparatus]

${ }^{1}$ Corresponding author.

E-MAIL pschedl@molbio.princeton.edu; FAX (609) 258-1028.

Article and publication are at http://www.genesdev.org/cgi/doi/10.1101/ gad.1316805. ing nuclear cycle 10, and, unlike the pole cells, they continue to divide synchronously until the end of nuclear cycle 14, when the embryo cellularizes and commences gastrulation.

Although Drosophila is unusual in that the early stages of development proceed in the absence of cells, many of the steps that need to occur by the time the cellular blastoderm is formed, such as the nuclear division cycles, transcriptional silencing, and the segregation of the germline and somatic lineages, involve mechanisms that are fundamental to most eukaryotes, and the genes that function in such processes are likely to be widely conserved. Recent studies on the yeast Schizosaccharomyces pombe and the trypanosome Trypanosoma brucei have shown that components of the RNAi machinery such as Argonaute-1 (Agol) and Dicer-1 (Dcr1) are critical for proper mitosis (DurandDubief and Bastin 2003; Volpe et al. 2003). In S. pombe lacking either Agol or Dcrl activity, centromere function is disrupted and there is a high incidence of lagging chromosomes in late anaphase, elevated rates of chromosome loss, and increased sensitivity to drugs that destabilize microtubules. The defects in centromere function appear to arise from a failure in heterochromatin assembly, and the centromeric repeats in the mutants are deficient in $\mathrm{K} 9$-methylated histone $\mathrm{H} 3$, have reduced levels of the heterochromatin-associated protein Swi6, and are not properly silenced (Hall et al. 2002; Volpe et al. 2002; Verdel et al. 2004). The RNAi machinery is also required for heterochromatin formation in Tetrahymena; however, in this and other protozoa, heterochromatin assembly plays a central role in the elimination of germline-specific (micronucleus) sequences in the newly formed somatic macronucleus following conjugation (Mochizuki and Gorovsky 2004).

We wondered whether the RNAi machinery might have comparable functions during early Drosophila embryogenesis. Mutations that are thought to eliminate the activity of the RNA-induced silencing complex (RISC) protein Ago-2 in Drosophila have recently been described (Okamura et al. 2004; Xu et al. 2004). While these mutants are defective in siRNA silencing, they are viable and fertile, suggesting that ago-2 might not function in processes that are critical for early embryonic development such as the assembly of centromeric heterochromatin, mitosis, or formation/segregation of the germline and somatic lineages. On the other hand, Yohn et al. (2003) have recently described mutations in three genes-1(3)malignant brain tumor, shakleton, and out of sync- - that have nuclear division, nuclear migration, and pole cell formation phenotypes. Remarkably, while the defects in nuclear division and migration in these mutants are sufficiently severe that they would be expected to have lethal consequences, most of the embryos manage to compensate and give rise to viable and fertile adults. Thus, it seemed possible that ago-2 might have functions during early embryogenesis that were missed in previous work.

\section{Results and Discussion}

Since the RNAi machinery has been implicated in the formation of centric heterochromatin and mitosis in unicellular organisms, we anticipated that a loss of ago-2 
activity might influence the rapid nuclear divisions in early fly embryos. To investigate this possibility, we compared wild-type and ago- $2^{414}$ mutant /Okamura et al. 2004) embryos stained with the DNA dye Hoechst. Several abnormalities were evident in ago-2 embryos. The spacing between nuclei in wild type is relatively constant during the early cleavage cycles, and the nuclei are distributed uniformly through the central region of the embryo (see Fig. 1A,C). In contrast, in ago-2 $2^{414}$ embryos, the distance from one nucleus to the next is quite variable, and nuclei are found clustered in some regions of the embryo while they are absent in other regions (see Fig. 1B,D). When the nuclei migrate out to the cortex in wild type during cycles $8-9$, they are spaced at regular intervals around the entire surface of the embryo (see Fig. 1E,G). This is not the case in ago- $2^{414}$ embryos. As shown in Figure $1 \mathrm{~F}$, many ago- $2^{414}$ nuclei fail to migrate to the surface, while others appear to reach the surface but then fall back into the interior of the embryo. Consequently, the nuclear distribution at the surface is quite irregular (Fig. 1H). In some regions there is a high concentration of nuclei, often linked together in "strings" of two, three, or four incompletely separated nuclei, while in other regions there are very few. There are also some very brightly stained nuclei that appear to have more than the normal complement of DNA, while other nuclei are smaller than normal (some are just small dots) and seem to be missing DNA. Defects in chromosome segregation are also evident prior to nuclear migration (Fig. 1D). Consistent with a requirement for ago-2 in the

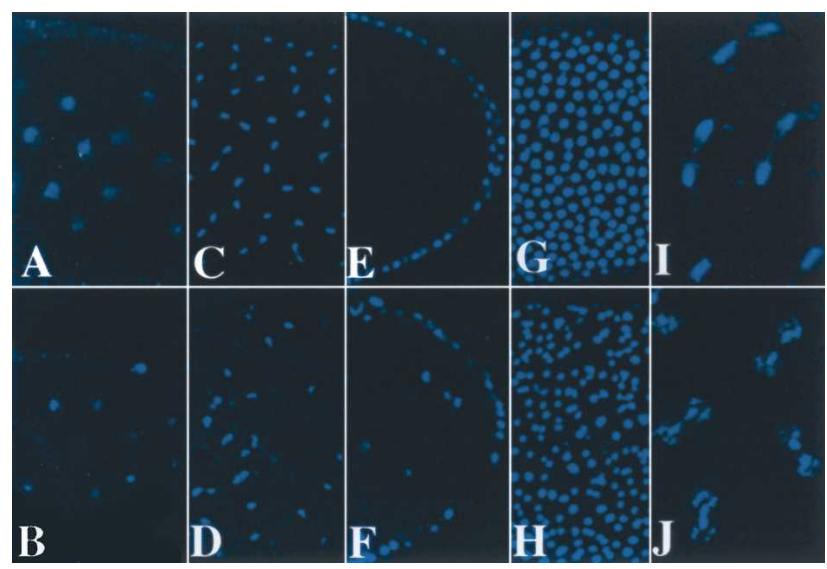

Figure 1. Disruption of early nuclear division cycles and cortical migration in ago-2 embryos. Hoechst-stained wild-type $(A, C, E, G, I)$ and ago-2 $(B, D, F, H, J)$ embryos. (A) Wild-type presyncytial blastoderm embryo showing regularly spaced nuclei in the interior of the embryo (the nuclear cloud stage). (B) Irregular nuclear spacing is seen in presyncytial blastoderm ago-2 embryos. Note that cortical migration appears to be prematurely initiated as several nuclei are at the periphery. (C) Wild-type early syncytial blastoderm embryo showing synchronously dividing nuclei during mitosis. (D) Early syncytial blastoderm ago-2 embryo showing asynchronous mitosis and uneven nuclear density. (E) Wild-type late syncytial blastoderm embryo showing interphase nuclei at the periphery when nuclear migration is completed. $(F)$ ago-2 late syncytial blastoderm embryo with interphase nuclei showing clear gaps at the periphery. Nuclei fallen inside of the embryo are seen. $(G)$ Surface view of wild-type late-stage syncytial blastoderm embryo showing uniform nuclear density. (H) ago-2 late syncytial blastoderm embryo showing uneven nuclear packing and mitotic asynchrony. (I) Wild-type embryo showing normal mitotic figures. $(J)$ Abnormal mitotic figures observed in ago-2 embryos. proper execution of the mitotic cycle, anomalous anaphase figures with incompletely condensed and irregularly positioned chromosomes are observed in the ago- $2^{414}$ mutant (cf. Fig. 1I,J).

Similar defects in nuclear division, migration, and spacing were observed for a second $a g o-2^{51 b}$ allele (Xu et al. 2004) and for trans-combinations between the two ago-2 mutations. In all three cases, the frequency of embryos showing at least some defect in nuclear division and/or nuclear migration was between $50 \%$ and $60 \%$. These abnormalities prompted us to re-examine the effects of ago-2 mutations on viability. We found that $10 \%-15 \%$ of ago- $2^{414}$ embryos failed to hatch, while nearly $40 \%$ of $a g o-2^{51 B}$ did not hatch. The viability of the trans-combination was intermediate between the homozygous mutants.

\section{Nuclear division cycles are asynchronous in ago-2 embryos}

To further characterize the progression of the nuclear division cycles in ago-2, we examined Histone $\mathrm{H} 3$ phosphorylation on Ser 10. In wild type, H3-Ser 10 phosphorylation can be detected nearly simultaneously in all nuclei when chromosome condensation commences at the onset of mitosis (Fig. 2A-C,G-I). High levels of H3 phospho-Ser 10 persist through mitosis and then disappear as the chromosomes start to decondense before the beginning of the next round of DNA synthesis. A variety of anomalies are evident in ago- $2^{414}$ embryos. In the early cleavage stage embryo shown in Figure 2D-F, these include fragmented nuclei (arrows in Fig. 2E) and nuclei that appear to have incompletely separated but are still entering mitosis (arrowhead in Fig. 2E). In the older embryo in Figure 2J-L, the most striking defect is the asynchrony in the nuclear division cycles (Fig. 2). Asynchrony can also sometimes be seen in incompletely separated nuclei (see arrowhead in Fig. 2J-L).

\section{Disruption of the mitotic spindle apparatus and an abnormal centrosome assembly in ago-2 embryos}

The nuclear division defects suggested that ago-2 may be important for the assembly or functioning of the mitotic spindle apparatus. In the wild-type embryo shown in Figure $3 \mathrm{~A}$, the chromosomes in each mitotic figure are aligned on the metaphase plate. Emanating from the chromosomes in opposite directions are microtubule arrays that converge at each centrosome. As illustrated by the metaphase figures in Figure $3 \mathrm{~B}$ and $\mathrm{C}$, nuclei that appear to be undergoing a normal mitosis are seen in ago- $2^{414}$ embryos. However, a variety of defects are also evident in more than half of the ago- $2^{414}$ embryos. Among the more striking are "orphan" centrosome pairs. While the duplicated centrosomes occasionally remain in close proximity (Fig. 3B, lower left), most appear to migrate to "opposite poles" (cf. arrowhead pairs). However, these orphan centrosome pairs are not associated with a mitotic spindle apparatus or with mitotic chromosomes, nor does there appear to be any nearby interphase nucleus. While other centrosome pairs appear to properly nucleate the mitotic spindle apparatus, the spindles are abnormally short and do not extend to the chromosomes or make connections with the centromeres (Fig. 3B, arrow). In these mitotic figures the chro- 

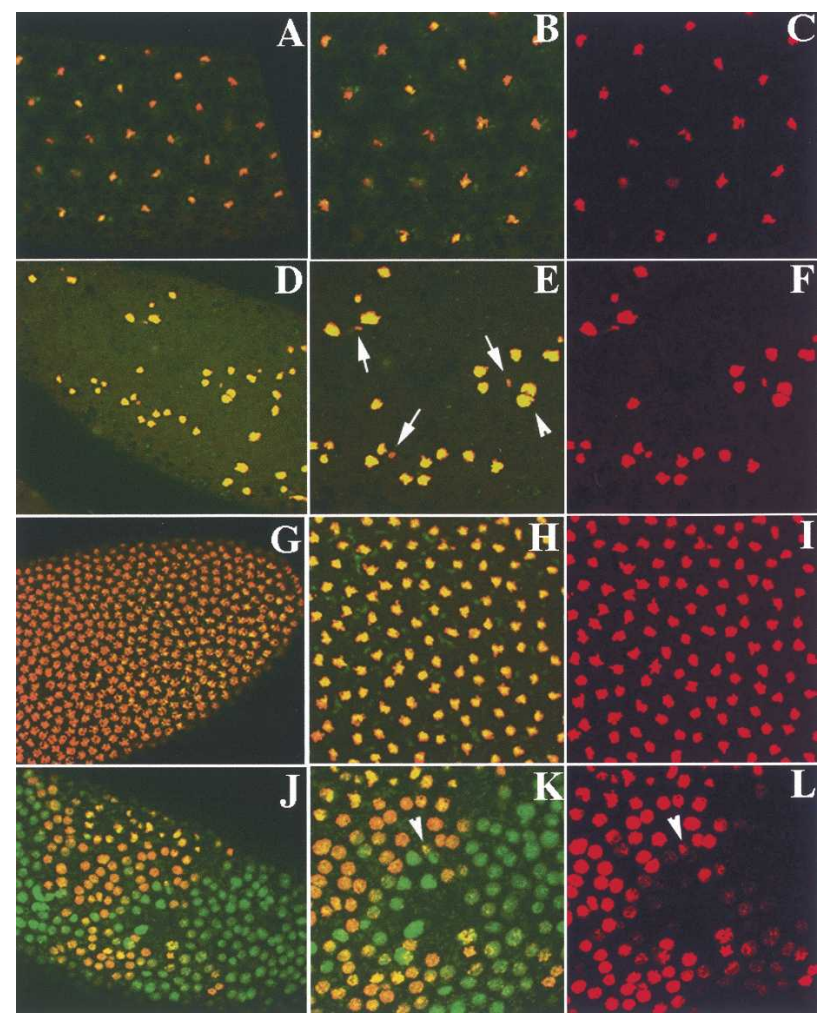

Figure 2. Mitotic asynchrony in early ago-2 embryos. Wild-type and $a g o-2$ embryos were stained with DNA dye, Hoechst (imaged in green), and phospho-histone $\mathrm{H} 3$ (pH3) antibody (imaged in red). (A Presyncytial blastoderm wild-type embryo showing synchronously dividing, evenly spaced nuclei that have $\mathrm{pH} 3$. A close-up from same embryo showing merged $(B)$ and $\mathrm{pH} 3(C)$ staining. $(D)$ Presyncytial blastoderm ago-2 embryo showing unevenly spaced and asynchronously dividing nuclei. A close-up from the same embryo shown in panels $E$ and $F$. Arrows in panel $E$ indicate asynchronous "micronuclei." $(G)$ Wild-type syncytial blastoderm embryo showing synchronously dividing nuclei. A close-up from the same embryo showing merged $(H)$ and $\mathrm{pH} 3(I)$ staining. $(J)$ ago-2 syncytial blastoderm embryo showing asynchronously dividing nuclei. $(K, L)$ A close-up from the same embryo. Note that only a subset of nuclei is positive for pH3. Arrowhead in panel $L$ indicates incompletely separated asynchronous nuclei.

mosomes often appear to be displaced from their normal position in the center of the metaphase plate. In other cases there are DNA bridges that extend between two adjacent mitotic figures (Fig. 3C, arrowhead). There are also "mitotic spindle" bridges that connect two different mitotic figures (Fig. 3D, arrow). Similar centrosomal and mitotic spindle defects are seen in $a g o-2^{51 b}$ embryos.

\section{Defects in centric and centromeric heterochromatin assembly in ago-2 embryos}

The mitotic defects in S. pombe and T. brucei lacking Ago activity have been attributed to a disruption in centromere function because of a failure to properly assemble centromeric heterochromatin and the flanking centric heterochromatin (Volpe et al. 2002, 2003; Durand-Dubief and Bastin 2003). It seemed possible that the abnormal nuclear division and the defects in the spindle apparatus evident in ago-2 embryos might also arise from a failure in the formation of heterochromatin across the centromeric repeats. Drosophila centromeric heterochromatin contains a centromere-specific histone $\mathrm{H} 3$ variant, called centromeric identifier (CID), that is critical for centromere function and assembly of the kinetochore (Blower and Karpen 2001). To examine centromeric heterochromatin, we probed ago- $2^{414}$ embryos with antibody against CID and centrosomin. During anaphase the chromosomes on the metaphase plate begin moving toward the two centrosomes. At this point in the mitotic cycle, the centromeres are located in close proximity to the centrosomes, while the chromosomal arms extend back toward the metaphase plate. As illustrated in Figure 4A, the centromeres can be visualized in wildtype chromosomes as several prominent dots of CID staining located near the base of the spindle apparatus in close proximity to each centrosome. While prominent CID dots can be seen on chromosomes throughout mitosis in wild-type embryos, this is not true in ago-2 414 embryos. In the ago- $2^{414}$ anaphase figure shown in Figure $4 \mathrm{~B}$, the centromeres of the chromosomes that have moved toward the centrosomes have CID dots; however, these are only weakly stained. There is also a chromosome that has remained behind that lacks CID protein. In the anaphase figure in Figure 4C, a subset of chromosomes have begun to migrate toward the centrosome on the right. On these chromosomes CID dots can be detected associated with the centromeres located near the centrosome, while the chromosomes that have remained on the metaphase plate do not have the characteristic

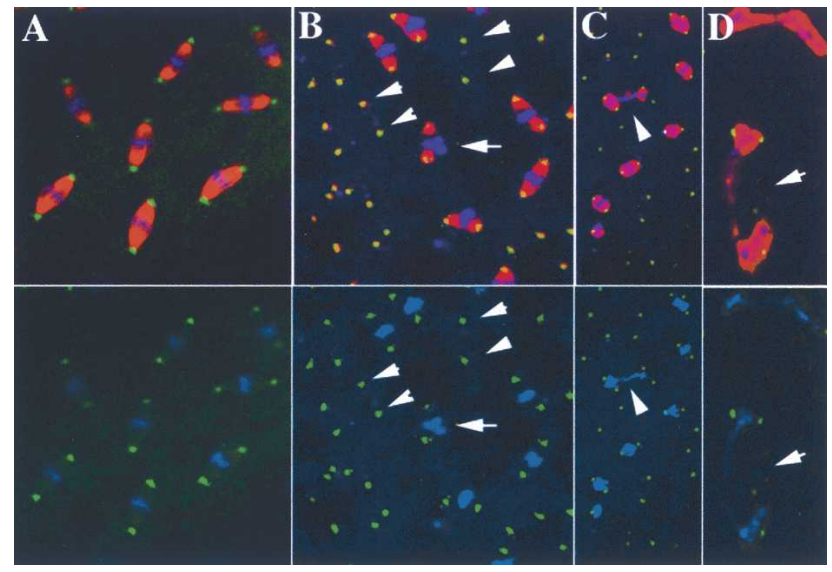

Figure 3. Defective centrosomal assembly and mitotic spindles in ago-2 embryos. Wild-type and ago- $2^{414}$ embryos stained with $\alpha$-tubulin (red) and centrosomin (green) antibodies and DNA dye, Hoechst (blue). Panels on the top are merged images while the panels on the bottom show just DNA and centrosomin. $(A)$ Wild-type embryo. The chromosomes aligned on the metaphase plate and microtubule arrays converging at each centrosome. $B-D$ show ago-2 embryos. $(B)$ A subset of nuclei appears to be undergoing normal mitosis; however, several abnormalities are also evident. These include "orphan" centrosome pairs, which have neither tubulin nor DNA/nuclei (arrowhead pairs). (Lower left) Some of the duplicated centrosomes seem to remain in close proximity. Also seen are abnormally short spindles that do not appear to extend to the chromosomes or make connections with the centromeres (arrow). (C) In some cases there are DNA bridges that extend between two adjacent mitotic figures (arrowhead). (D) There are also "mitotic spindle" bridges that connect two different mitotic figures. The arrow indicates two mitotic spindles that are connected by tubulin and DNA. There appear to be two centrosomes associated with each mitotic figure; however, the mitotic spindle does not converge on one of these centrosomes. 

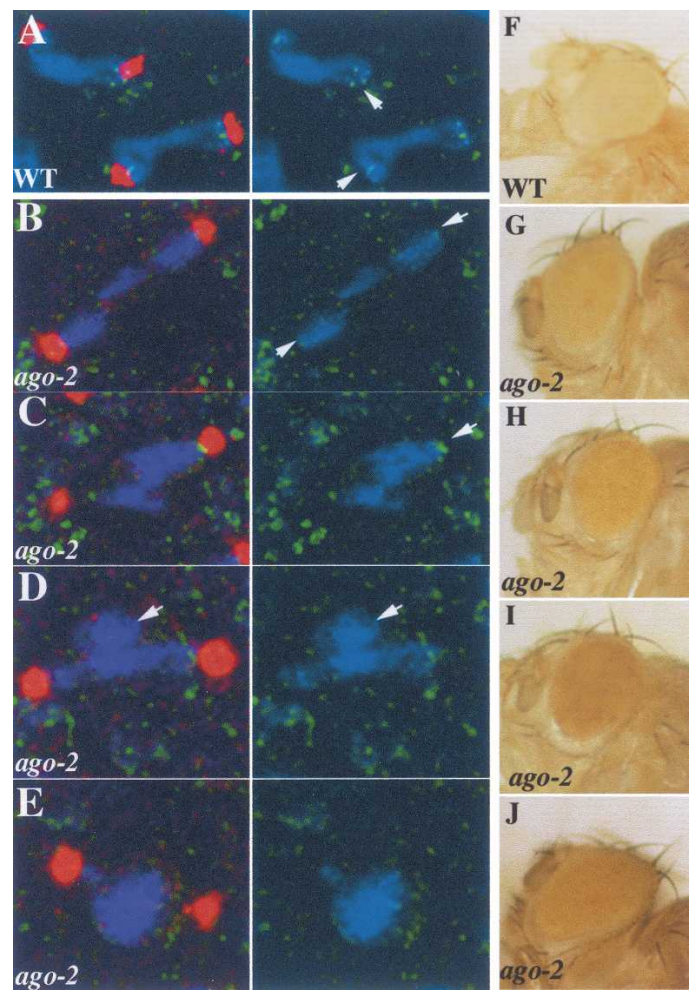

$\mathbf{H}$
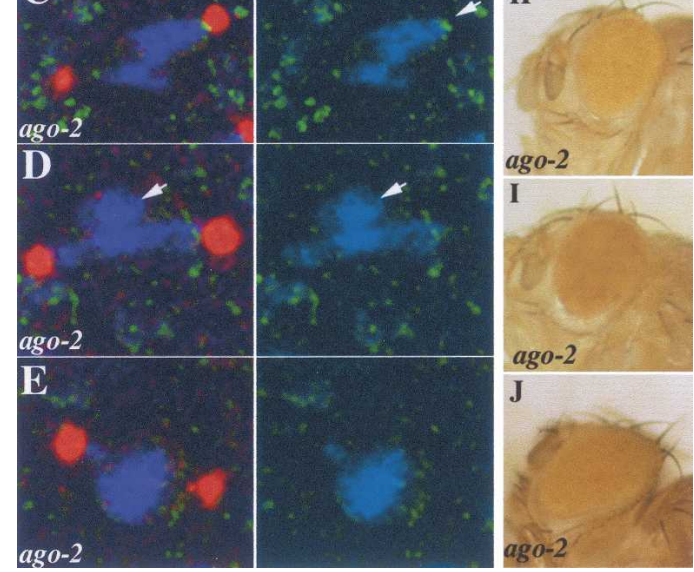

Figure 4. ago-2 is required for the localization of CID to centromeres and for heterochromatic silencing of mini-white transgenes $(A-D)$ Panels on top show mitotic figures in wild-type and ago-2 embryos, respectively, stained with Hoechst DNA dye (blue), CID (green), and Centrosomin (red) antibody. CID "spots" are localized near the centrosomes in wild-type mitotic figures (see arrows in accompanying panel). In ago-2 embryos, CID staining is weak (arrows in $B$ ), on only the chromosomes that move toward the centrosomes (arrow in $C$ ), absent from chromosomes that remain at the metaphase plate $(D, E)$. F-J show the eye of wild-type or ago-2/+flies carrying a mini-white transgene inserted into centric heterochromatin of the second chromosome (39C-4). Male flies homozygous for the mini-white insert were mated to ago- $2^{414}$ females. Note that the four ago-2/+ flies have different eye colors; however, the pigmentation is relatively uniform across the eye (and the same in the other eye) and with the exception of a few ommatidia in $H$ and $I$, there is little evidence of variegation.

CID dots. In the mitotic figures in Figure 4E and F, CID protein cannot be detected in the $a g 0-2^{414}$ chromosomes that have remained on the metaphase plate (see arrowheads).

Flanking the centromere is the centric heterochromatin, which is marked by elevated levels of histone H3 methylated on Lys 9 (H3 meK9) and by the Swi6 homolog, Heterochromatin protein 1 (HP-1) (Kellum and Alberts 1995; Kellum et al. 1995). We found that association of HP-1 with centric heterochromatin (Supplementary Fig. 1) is abnormal in ago- $2^{414}$ precellular blastoderm embryos. There also appear to be defects in K9 methylation (data not shown).

ago-2 is required for heterochromatic silencing early in development

If ago-2 is required for the formation of centromeric/ centric heterochromatin then ago-2 mutations should suppress silencing of mini-white transgenes inserted into pericentric heterochromatin (Cryderman et al. 1998). Males carrying mini-white transgenes inserted into pericentric regions of the second, third, or fourth chromosomes were mated to ago-2 $2^{414}$ females, and the eye color phenotype of $a g o-2^{414} /+$ adults was examined. For inserts on all three chromosomes, we found that mini-white expression is up-regulated in $\sim 7 \%$ of the ago- $2^{414} /+$ flies (Fig. 4, cf. F and G-J). Significantly, the pattern of suppression points to a very early role for ago-2 in the establishment of silenced heterochromatin domains. Thus, while the eye color varies from one fly to the next, in the flies showing suppression, both eyes typically have very similar pigmentation. Moreover, instead of a highly variegated phenotype, the pigmentation is (with the exception of a few ommatidia) usually quite uniform across each eye. These results suggest that maternally derived ago-2 activity is required for establishing silenced heterochromatin domains in the paternal chromosomes at a point prior to nuclear migration.

\section{ago-2 embryos have defects in the actin-myosin cytoskeleton}

The occurrence of "orphaned" centrosomes prompted us to examine the cytoskeleton in ago-2 embryos with antibodies against Anillin, a component of the actin-myosin contractile ring, and Peanut, a septin (Fares et al. 1995; Foe et al. 2000). During interphase in wild-type Drosophila nuclear cycle 13-14 blastoderm embryos, cortical actin forms a cap on the apical surface of the embryo above the nucleus. Anillin is localized just underneath the actin cap in a ring that surrounds the nucleus, and in surface views (Fig. 5A) it gives a quite regular mesh-like appearance. In $\sim 50 \%$ of the $a g o-2^{414}$ embryos this regular lattice is replaced by a broken network with irregularly shaped contractile rings that vary in thickness from one part of the ring to the next (Fig. $5 \mathrm{~B})$. Some of the anillin rings appear to contain multiple nuclei, while other rings have neither nuclei nor DNA. Similar irregularities are also observed in the Peanut lattice/network (Supplementary Fig. 2).

The anillin lattice is not present in wild-type embryos during earlier nuclear cycles, and instead there is a donut-like structure around each nucleus (Fig. 5C,E). While the anillin "donut" is also seen around many nuclei in ago-2 embryos of a similar age, it is often absent altogether (Fig. 5D,F). In other cases, the DNA seems to surround the "donut-hole" (see arrows in Fig. 5F), instead of being the "donut-hole" as it is in wild type. Anillin can also be seen concentrated inside the nucleus (Fig. 5F, arrowheads). Interestingly, when we observe defects in the anillin donuts, they are typically associated with abnormalities in nuclear morphology or with fragmented or otherwise anomalous chromosomes (Fig. 5D, arrow and arrowheads). This finding would suggest that there is a link between the assembly and functioning of the mitotic apparatus and the proper organization of actin-myosin cytoskeleton. For example, the assembly of the anillin donuts/contractile rings may depend on the presence of a functional mitotic spindle and/or the reformation of the nuclear envelope at the end of mitosis. When the mitotic spindle is absent or disrupted, or if the nuclear envelope does not reform because of chromosome fragmentation, this could prevent the assembly of the regular anillin donuts/contractile rings. 


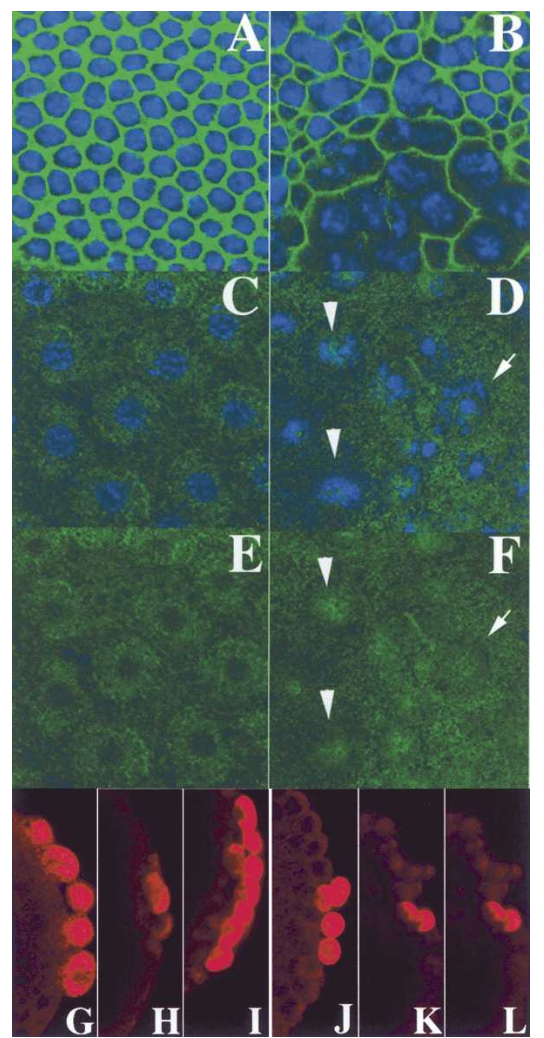

Figure 5. Anillin localization and pole cell formation are disrupted in ago-2 embryos. Wild-type $(A, C, E)$ and ago-2 $(B, D, F)$ embryos were stained with Hoechst DNA dye (blue) and Anillin antibody (green). $A$ and $B$ are surface views of cellularizing blastoderm embryos. An organized and regular anillin network is evident in wild type $(A)$, while this network is disrupted in ago-2 $(B)$. $C$ and $E$ show the annillin "donuts" in a wild-type presyncytial blastoderm embryo. $D$ and $F$ show that the organization of the anillin "donuts" and of the DNA is disrupted in a presyncytial ago-2 embryo. Arrowheads indicate colocalization of anillin and DNA, while the arrow shows a "nucleus" in which part of the DNA forms the "donut." $G-L$ show pole cells: 0-4-h ago-2 and wild-type embryos were fixed and stained with Vasa antibody (imaged in red) and DNA dye, Hoechst (not shown). $(G)$ Wild-type presyncytial stage embryo showing pole buds. (H) ago-2 presyncytial stage embryo showing a reduced number of pole buds. (I) Wild-type syncytial blastoderm showing several pole cells in the plane of focus. $(J-L)$ Three examples of ago-2 syncytial blastoderm embryos showing a reduced number of pole cells.

\section{ago-2 is required for pole cell formation}

Since proper nuclear migration is necessary for pole cell formation (Yohn et al. 2003), we counted the number of pole cells at the syncytial blastoderm stage (nuclear cycles 12-13) in ago-2 ${ }^{414}$ embryos stained with Vasa antibody and counterstained with the DNA dye Hoechst. We found that the total number of pole cells in ago- $2^{414}$ (eight per embryo; $n=20$ ) is also much less than wild type (20 per embryo; $n=15$ ). As illustrated in Figure 5J and $\mathrm{K}$, many ago- $2^{414}$ embryos had only two or three pole cells, and the pole cells were often not positioned correctly at the very posterior or were separated from each other by somatic cells. There was also a great deal of variability in the number of pole cells in the ago-2 mutant that is not evident in wild type. While some ago-2 $2^{414}$ embryos had only two or three pole cells, there were a few that had near wild-type numbers. A similar, though not quite as dramatic reduction in pole cell number was observed for a second allele, ago-2 ${ }^{51 B}$ (12 per embryo). The reduction in the number of pole cells can be traced back to nuclear cycle 8-9 when migrating nuclei first enter the posterior pole plasm. As illustrated in Figure 5H, several nuclear cycle 8-9 ago-2414 embryos have fewer pole buds than wild type (Fig. 5G).

Two questions are posed by our findings. The first is the relationship between the phenotypes seen in early embryos and ago-2 function. Although we cannot exclude the possibility that ago-2 participates in several distinct pathways, we suspect that many of the phenotypes are attributable either directly or indirectly to a requirement for $a g o-2$ in the proper execution of the nuclear division cycles. In principle, ago-2 could impact the division cycles by promoting the degradation and/or translational repression of $\mathrm{mRNAs}$ encoding factors that somehow impede the cleavage cycles. However, since the RNAi machinery has been implicated in the establishment of heterochromatin domains (cf. Pal-Bhadra et al. 2004; Verdel et al. 2004), an alternative possibility is that ago-2 is important for generating the appropriate chromatin organization of cleavage-stage chromosomes. In S. pombe, deletion of Ago-1 (the only Ago gene) disrupts the formation of transcriptionally silenced heterochromatin domains near the centromeres, and this impairs the assembly/functioning of the centromeres (Volpe et al. 2002, 2003). Most of the abnormalities in the division cycles and mitotic apparatus evident in ago-2 embryos could also be explained by perturbations in the assembly/functioning of the centromeres. Probably the most compelling evidence in favor of this hypothesis are the defects in CID association with ago-2 chromosomes. We observe dividing nuclei in which a subset of chromosomes or in some cases all of the chromosomes have little or no detectable CID. Strikingly, in these anaphase figures the chromosomes that have little CID typically remain on the metaphase plate, while the chromosomes that have CID are seen to migrate toward the centrosomes. The effects of ago-2 are not limited to centromeric heterochromatin. We found that the silencing of paternally inherited mini-white transgenes inserted into centric heterochromatin can be suppressed by reducing ago-2 activity in the mother. Significantly, the pattern of suppression-a nearly uniform level of pigmentation that is the same in both eyes-argues that ago-2 activity is required very early in development during the initial "establishment" of silenced heterochromatin. Consistent with an early role in heterochromatin assembly, we also found abnormalities in HP1 localization. While our results do not provide insights into how Ago-2 might actually function in this process, the idea that the RNAi machinery plays some special role in the de novo assembly of centromeric/centric heterochromatin during the embryonic nuclear division cycles is supported by the finding that rasiRNAs are highly enriched in early embryos but not at later stages of development (Aravin et al. 2003).

The second question is why do the ago-2 mutants show incomplete penetrance and expressivity. One reason may be the plasticity of early embryos that enables cells in regions of the embryo that are normal to compensate for cells in regions that have major defects. Consistent with this suggestion, Yohn et al. (2003) described mutations in three genes, 1(3)malignant brain tumor, shakleton, and out of sync, that have a range of nuclear 
division and migration phenotypes similar to those seen in the two ago-2 alleles. In spite of the defects observed in these mutants, Yohn et al. (2003) found that most of the embryos hatch and go on to form fertile adults. Another reason is that the two mutants we examined may not be complete nulls. Consistent with this possibility, a low level of Ago-2 protein was detected in mutant embryos (G. Deshpande, unpubl.). Finally, there could be other partially redundant mechanisms that ensure the formation and/or maintenance of centromeric/centric heterochromatin and functional centromeres. This idea is supported by the studies of Pal-Bhadra et al. (2004), who found that like ago-2, mutations in two other ago-2-like proteins, piwi and aubergine, suppress heterochromatic silencing of mini-white transgenes and disrupt the localization of heterochromatin proteins HP1 and HP2. Thus, it is possible that either of these two genes or perhaps other components of the RNAi machinery are able to substitute for ago-2 and promote the establishment and/or maintenance of heterochromatin domains and functional centromeres in early embryos.

\section{Materials and methods}

Strains and culturing

Flies were grown on a standard medium at $25^{\circ} \mathrm{C}$ unless otherwise noted.

\section{Immunohistochemistry}

The embryo stainings were performed essentially as described in Deshpande et al. (1995). Embryos were usually fixed in paraformaldehyde/ heptane mix for 15-20 min and vitelline membranes were subsequently removed by shaking in methanol upon removal of paraformaldehyde. In the case of anti-tubulin antibodies, embryos were fixed just in methanol/ heptane mix for an hour. The following antibodies were used at a prescribed dilution in a standard immunohistochemical analysis: Anti-Peanut (mouse monoclonal from Developmental Hybridoma Bank, used at 1:20), anti-Anillin (rabbit polyclonal used at 1:500, a gift from C. Field, Harvard Medical School, Boston, MA), anti-Phosphohistone H3 (rabbit polyclonal used at 1:500; Upstate Biotechnology), anti-CID (chicken polyclonal used at 1:100; a gift from G. Karpen, Lawrence Berkeley National Laboratory, Berkeley, CA), anti-Centrosomin (rabbit polyclonal used at 1:500; a gift from T. Kaufman, Indiana University, Bloomington, IN), anti-HP1 (rabbit polyclonal used at 1:500; a gift from R. Kellum, University of Kentucky, Lexington, KY), and anti-Vasa (rabbit polyclonal antibody that was preabsorbed against wild-type [WT] embryonic samples and was subsequently used at 1:500 dilution; a gift from P. Lasko, McGill University, Montreal, Canada). Anti- $\beta$-galactosidase (rabbit) antibody was purchased from Kappel and was used at 1:1000 dilution after preabsorbing it against wild-type embryonic sample. Unless otherwise specified, we examined at least 25 embryos of the relevant stage and found that the frequency of various defects in the ago-2 mutant embryos was $\sim 50 \%$.

\section{Acknowledgments}

We thank F.B. Gao, A. Muller, T. Schupbach, H. Siomi, L. Wallrath, and the Bloomington Stock Center for flies, and G. Karpen, T. Kaufman, P. Lasko, R. Kellum, and E. Wieschaus for antibodies. We are particularly grateful to A. Muller and M. Welte for communicating results prior to publication, and to I. Clark, G. Shanower, M. Goldberg, and D. Marks for advice and helpful discussions during the course of this work. We also thank J. Goodhouse for help with confocal microscopy and Gordon Grey for fly food. This work was supported by an NIH grant to P.S.

\section{References}

Aravin, A.A., Lagos-Quintana, M., Yalcin, A., Zavolan, M., Marks, D., Snyder, B., Gaasterland, T., Meyer, J., and Tuschl, T. 2003. The small RNA profile during Drosophila melanogaster development. Dev. Cell 5: 337-350.
Baker, J., Theurkauf, W.E., and Schubiger, G. 1993. Dynamic changes in microtubule configuration correlate with nuclear migration in the preblastoderm Drosophila embryo. J. Cell Biol. 122: 113-121.

Blower, M.D. and Karpen, G.H. 2001. The role of Drosophila CID in kinetochore formation, cell-cycle progression and heterochromatin interactions. Nat. Cell Biol. 3: 730-739.

Cryderman, D.E., Cuaycong, M.H., Elgin, S.C.R., and Wallrath, L.L. 1998. Characterization of sequences associated with position-effect variegation at pericentric sites in Drosophila heterochromatin. Chromosoma 107: 277-285.

Deshpande, G., Stukey, J., and Schedl, P. 1995. scute (sis-b) function in Drosophila sex determination. Mol. Cell. Biol. 15: 4430-4440.

Durand-Dubief, M. and Bastin, P. 2003. TbAGO1, an Argonaute protein required for RNA interference, is involved in mitosis and chromosome segregation in Trypanosoma brucei. BMC Biol. 1: 2.

Fares, H., Peifer, M., and Pringle, J.R. 1995. Localization and possible functions of Drosophila septins. Mol. Biol. Cell 6: 1843-1859.

Foe, V.E., Field, C.M., and Odell, G.M. 2000. Microtubules and mitotic cycle phase modulate spatiotemporal distributions of F-actin and myosin II in Drosophila syncytial blastoderm embryos. Development 127: 1767-1787.

Hall, I.M., Shankaranarayana, G.D., Noma, K., Ayoub, N., Cohen, A., and Grewal, S.I. 2002. Establishment and maintenance of a heterochromatin domain. Science 297: 2232-2237.

Kellum, R. and Alberts, B.M. 1995. Heterochromatin protein 1 is required for correct chromosome segregation in Drosophila embryos. J. Cell Sci. 108: 1419-1431.

Kellum, R., Raff, J.W., and Alberts, B.M. 1995. Heterochromatin protein 1 distribution during development and during the cell cycle in Drosophila embryos. J. Cell Sci. 108: 1407-1418.

Mochizuki, K. and Gorovsky, M.A. 2004. Small RNAs in genome rearrangement in Tetrahymena. Curr. Opin. Genet. Dev. 14: 181-187.

Okamura, K., Ishizuka, A., Siomi, H., and Siomi, M.C. 2004. Distinct roles for Argonaute proteins in small RNA-directed RNA cleavage pathways. Genes \& Dev. 18: 1655-1666.

Pal-Bhadra, M., Leibovitch, B.A., Gandhi, S.G., Rao, M., Bhadra, U., Birchler, J.A., and Elgin, S.C. 2004. Heterochromatic silencing and HP1 localization in Drosophila are dependent on the RNAi machinery. Science 303: 669-672.

Sullivan, W., Fogarty, P., and Theurkauf, W. 1993. Mutations affecting the cytoskeletal organization of syncytial Drosophila embryos. Development 118: 1245-1254.

Verdel, A., Jia, S., Gerber, S., Sugiyama, T., Gygi, S., Grewal, S.I., and Moazed, D. 2004. RNAi-mediated targeting of heterochromatin by the RITS complex. Science 303: 672-676.

Volpe, T.A., Kidner, C., Hall, I.M., Teng, G., Grewal, S.I., and Martienssen, R.A. 2002. Regulation of heterochromatic silencing and histone H3 lysine-9 methylation by RNAi. Science 297: 1833-1837.

Volpe, T., Schramke, V., Hamilton, G.L., White, S.A., Teng, G., Martienssen, R.A., and Allshire, R.C. 2003. RNA interference is required for normal centromere function in fission yeast. Chromosome Res. 11: 137-146.

von Dassow, G. and Schubiger, G. 1994. How an actin network might cause fountain streaming and nuclear migration in the syncytial Drosophila embryo. J. Cell Biol. 127: 1637-1653.

Williamson, A. and Lehmann, R. 1996. Germ cell development in Drosophila. Annu. Rev. Cell Dev. Biol. 12: 365-391.

Xu, K., Bogert, B.A., Li, W., Su, K., Lee, A., and Gao, F.B. 2004. The fragile X-related gene affects the crawling behavior of Drosophila larvae by regulating the mRNA level of the DEG/ENaC protein pickpocket1. Curr. Biol. 14: 1025-1034.

Yohn, C.B., Pusateri, L., Barbosa, V., and Lehmann, R. 2003. 1(3)malignant brain tumor and three novel genes are required for Drosophila germ-cell formation. Genetics 165: 1889-1900. 


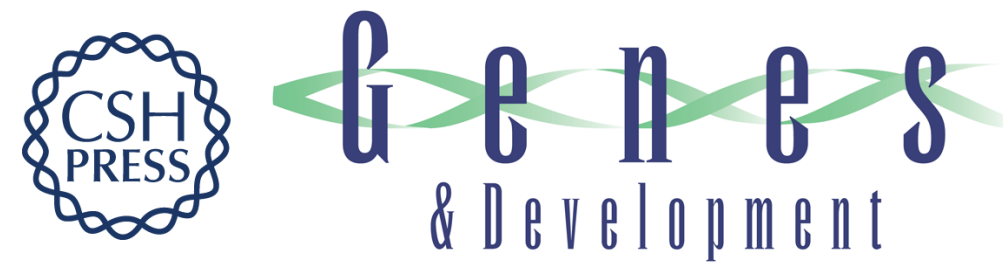

\section{Drosophila argonaute-2 is required early in embryogenesis for the assembly of centric/centromeric heterochromatin, nuclear division, nuclear migration, and germ-cell formation}

Girish Deshpande, Gretchen Calhoun and Paul Schedl

Genes Dev. 2005, 19:

Access the most recent version at doi:10.1101/gad.1316805

Supplemental http://genesdev.cshlp.org/content/suppl/2005/07/12/19.14.1680.DC1

Material

References This article cites 22 articles, 14 of which can be accessed free at: http://genesdev.cshlp.org/content/19/14/1680.full.html\#ref-list-1

License

Email Alerting Receive free email alerts when new articles cite this article - sign up in the box at the top Service right corner of the article or click here.

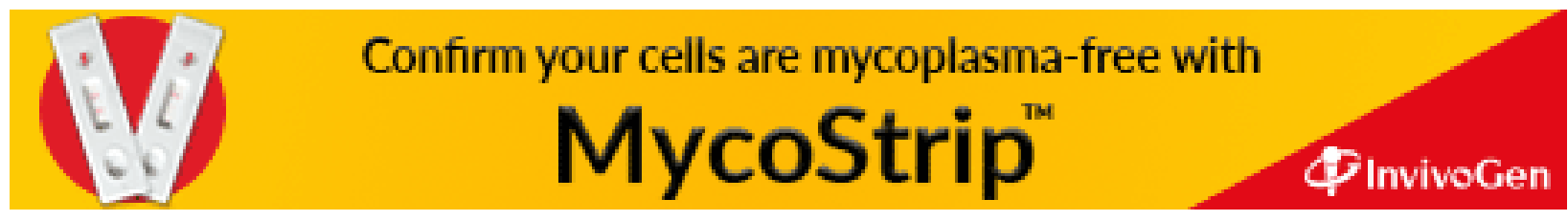

